\title{
Announcement: Howard Rosenbrock Prize 2016
}

\author{
Miguel F. Anjos 1
}

(C) Springer Science+Business Media, LLC 2017

I am delighted to announce that the recipients of the Howard Rosenbrock Prize 2016 are

\section{Taedong Kim and Stephen J. Wright}

of the Department of Computer Sciences, University of Wisconsin-Madison.

Their winning article is entitled

An $\mathbf{S} \ell_{1} \mathbf{L P}$-active set approach for feasibility restoration in power systems (Kim and Wright 2016).

The citation reads:

This article develops novel applied mathematical techniques to solve a crucial engineering problem. Namely, the authors consider the problem of minimizing the amount and number of load and generation adjustments in a power network that is affected by an attack or disturbance. The nonlinear nature of the physical laws governing the distribution of power in the network makes this problem very challenging to solve. To address this, the authors propose a novel use of nonsmooth penalty functions, sequential linear programming techniques, and active-set heuristics. Besides finding the optimal response to a network attack or disturbance, this approach allows the identification of network vulnerabilities. Given the importance of power distribution networks, and the reliability challenges faced by these networks due to security, climate, or other potential threats, this work constitutes an excellent contribution towards the continued development and operation of more reliable networks.

Miguel F. Anjos

anjos@stanfordalumni.org

1 Inria International Chair and NSERC-Hydro-Québec-Schneider Electric Senior Industrial Research Chair on Optimization for Smart Grids, GERAD and Polytechnique Montréal, C.P. 6079, Succ. Centre-ville, Montreal, QC H3C 3A7, Canada 
The Howard Rosenbrock Prize is sponsored by Springer, and the winners receive a cash award of USD $\$ 500$ and individual plaques.

Taedong Kim (left) and Stephen Wright
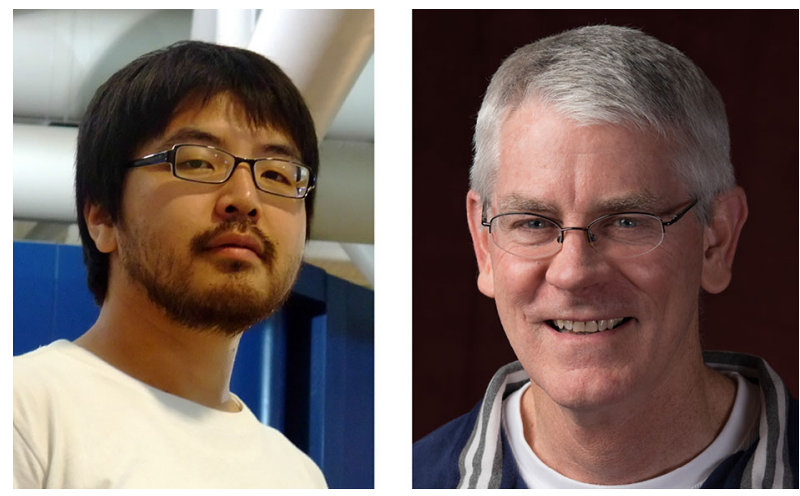

OPTE is a multidisciplinary journal that provides a forum where engineering researchers can obtain information about new developments in optimization, and optimization researchers can learn about the successes of optimization in engineering fields. The prize is named after Howard Rosenbrock, who embodied this bridging of the gap between optimization and engineering (Anjos 2015).

In 2016 the winning paper was selected by a committee of OPTE editors, chaired by Shabbir Ahmed, and composed of Marina Epelman, Roy Kwon, Makoto Ohsaki, and Luis Zuluaga. I am grateful to them for agreeing to contribute their time and expertise to the selection process.

\section{References}

Kim T, Wright SJ (2016) An $\mathrm{S} \ell_{1}$ LP-active set approach for feasibility restoration in power systems. Optim Eng 76(2):385-419

Anjos MF (2015) Announcement: Inaugural Howard Rosenbrock Prize. Optim Eng 16(3):507-509 